\title{
PAPER
}

\section{Lifespan differences in cortical dynamics of auditory perception}

\section{Viktor Müller, ${ }^{1,2}$ Walter Gruber, ${ }^{3}$ Wolfgang Klimesch ${ }^{3}$ and Ulman Lindenberger ${ }^{1,2}$}

\author{
1. Center for Lifespan Psychology, Max Planck Institute for Human Development, Berlin, Germany \\ 2. School of Psychology, Saarland University, Germany \\ 3. Department of Physiological Psychology, University of Salzburg, Austria
}

\begin{abstract}
Using electroencephalographic recordings (EEG), we assessed differences in oscillatory cortical activity during auditory-oddball performance between children aged 9-13 years, younger adults, and older adults. From childhood to old age, phase synchronization increased within and between electrodes, whereas whole power and evoked power decreased. We conclude that the cortical dynamics of perceptual processing undergo substantial reorganization from childhood to old age, and discuss possible reasons for the inverse relation between age trends in phase synchronization and power, such as lifespan differences in neural background activity, or a lifespan shift from rate coding in children to temporal coding in adults.
\end{abstract}

\section{Introduction}

How do oscillatory circuitry and synchronicity patterns during perception change from childhood to old age? Behavioral and physiological evidence suggests that interactions between maturation and learning from childhood to adulthood lead to increasing cortical differentiation and integration (Nelson \& Luciana, 2001), and that senescent changes from adulthood to old age result in dedifferentiation and reduced cortical specialization of neural cell assemblies (Baltes \& Lindenberger, 1997; Li, Lindenberger \& Sikström, 2001; Park, Polk, Park, Minear, Savage \& Smith, 2004). However, surprisingly little is known about lifespan changes in basic neuronal mechanisms, such as the coding of sensory events during perception and attentional processing. Here, we examine the cortical representation of sensory events in children of different ages, younger adults, and older adults using the auditory oddball task, an established paradigm to study attentional mechanisms across the lifespan (Polich, 1996, 1997; Yordanova \& Kolev, 1997, 1998).

The electroencephalogram (EEG) offers a rich source of information about neural coding dynamics (Nunez, 1995). Here, we derived four different spectral power and synchronization measures from the EEG that capture complementary aspects of the brain's neuronal activity (Gruber, Klimesch, Sauseng \& Doppelmayr, 2005; Lachaux, Rodriguez, van Quyen, Lutz, Martinerie \& Varela, 2000; Simoes, Jensen, Parkkonen \& Hari, 2003; Varela, Lachaux, Rodriguez \& Martinerie, 2001).
Different events or stimuli can induce time-locked or phase-locked changes in brain electrophysiological activities partially reflected in ERPs (event-related potentials), that is, composite time-averaged waveforms (Cacace \& McFarland, 2003). This proposition is valid even if strong assumptions about ERP analysis, such as the status of ongoing but stimulus-unlocked activity as processing-irrelevant noise, are called into question (cf. Makeig, Westerfield, Jung, Enghoff, Townsend, Courchesne \& Sejnowski, 2002; Pfurtscheller \& Lopes da Silva, 1999). ERPs reflect invariant changes of ongoing EEG activity evoked by the stimulus. Similarly, evoked power (EP) reflects stimulus-locked EEG activity in the time-frequency domain, as induced changes in EEG activity unlocked to the stimulus are eliminated through averaging. While evoked power is an amplitude estimate of the EEG averaged over trials, or the time-frequency rendition of ERPs, whole power (WP) calculates the power of each single trial before averaging (see Methods) and, thus, also comprises activity that is not stimulus-locked.

While ERPs as well as power measures are amplitude estimates, the other two measures used in this study, Phase Locking Index (PLI) and Phase Coherence (PC), are estimates of phase synchronization across trials (Gruber et al., 2005; Klimesch, Schack, Schabus, Doppelmayr, Gruber \& Sauseng, 2004). PLI reflects invariance of phases under single electrodes and PC emphasizes the constancy of phase differences between two electrodes across trials. Thus, PLI and PC are direct measures of temporal synchronization within and 
between electrode sites. However, note that we also refer to the power measures (i.e. EP and WP) in terms of synchronization, as they reflect neural synchronization or synchronous cell activity under a given electrode (local synchronization; see Pfurtscheller \& Lopes da Silva, 1999; Srinivasan, Russell, Edelman \& Tononi, 1999; Varela et al., 2001). In sum, both power and phase measures represent synchronization mechanisms used by the brain for information processing, and reflect the neural dynamics and the power or energy costs of involved cell assemblies.

Neuronal oscillations in mammalian cortical networks can be observed across a wide frequency range from approximately 0.05 to $500 \mathrm{~Hz}$ (Buzsáki \& Draguhn, 2004). The network oscillations may functionally bias stimulus selection, support transient bindings of neuronal assemblies, and facilitate synaptic plasticity. Theta oscillations are particularly prominent, with possible functional roles covering a wide range of behavior from arousal, attention and memory to orienting reflex, conditioning and learning, including different binding and information processing mechanisms (Buzsáki, 2005). Similarly, enhanced oscillatory activity in the delta frequency range during cognitive tasks is often considered as an indicator of attentional task demands (Harmony, Fernandez, Silva, Bernal, Diaz-Comas, Reyes, Marosi, Rodriguez \& Rodriguez, 1996; McEvoy, Pellouchoud, Smith \& Gevins, 2001). In contrast to delta and theta frequencies, alpha and beta rhythms tend to decrease or desynchronize in perceptual decision and memory tasks (Pfurtscheller \& Lopes da Silva, 1999), though event-related alpha desynchronization could not be found in several auditory tasks (Karrasch, Krause, Laine, Lang \& Lehto, 1998; Krause, Lang, Laine, Kuuisto \& Pörn, 1996). In a study using an auditory oddball paradigm (Cacace \& McFarland, 2003), synchronized EEG activity in delta, theta and alpha frequency bands and event-related desynchronization at the beta frequency were observed.

Evidence from animal studies suggests that neural activity during development is more competitive, stabilizing coincident inputs and weakening or removing non-coincident inputs relative to adults, who show collaborative or stabilized activity (e.g. McAllister, Katz \& Lo, 1999). In humans, using an auditory oddball task, Yordanova and Kolev $(1997,1998)$ found lower phaselocking and higher power in 6- to 11-year-old children than in young adults for both theta and alpha frequencies. Phase-locking was not only lower in children relative to young adults but also showed a continuous increase from 6 to 11 years. At the other end of the lifespan, differences in phase synchronization between younger and older adults have not so far been examined. However, some studies investigated changes in EEG power during normal aging. Generally, older adults show reduced spectral power in the delta and theta frequency bands during cognitive tasks (Duffy, McAnulty \& Albert, 1993; Marciani, Maschio, Spanedda, Caltagirone, Gigli \& Bernardi, 1994; McEvoy et al., 2001; Oken \& Kaye,
1992), probably indicating reduced attention, memoryrelated processes, or both (Harmony et al., 1996; McEvoy et al., 2001). However, standard spectral power measures used in previous studies have low temporal resolution and did not distinguish between phase-locked and induced power components. These two components have distinct functional meanings (Bastiaansen \& Hagoort, 2003; Tallon-Baudry \& Bertrand, 1999) and should, therefore, be separated. Also, the relations between aging-associated decrements in spectral power and stimulus-related phase locking have remained unclear. Separation of amplitude and phase components permits a better understanding of neural mechanisms involved in stimulus processing and neural representation. Here, we use a complex Gabor transform with high temporal resolution that allows for the separation of relevant spectral components.

We present data from four age groups (younger children, older children, younger adults, and older adults) obtained with an auditory oddball task under passive listening (unattended) and active counting (attended) conditions. Our expectations were that amplitude (i.e. power) and phase synchronization would change with age in different ways: phase synchronization, which reflects maturation and learning, should increase, while power, especially whole power, as an estimate of energy spectral density or resource allocation, should decrease with age. To aid the functional interpretation of synchronization phenomena, we also examined the relation between synchronization measures and behavioral performance on cognitive tests indicating perceptual speed. We expected perceptual speed scores to show a positive relation to synchronization, especially in young adults.

\section{Method}

\section{Participants}

All participants were volunteers and were recruited through announcements in Saarland schools (Gymnasiums) and Saarland University. The older adults were either auditors at Saarland University, participants in other continuing education programs, or both. For participation in the study, all subjects were paid 7.5 Euro per hour. All the subjects were right-handed, had no reported history of head or neurological disorders, and none were on medication. Of the participating individuals, five younger children, one older child, and one older adult were excluded from data analysis because they reported numbers of odd stimuli in attended conditions (see below) that deviated more than 3 digits in either direction from the correct number. Thus, the effective sample consisted of 24 younger children (YC, mean age $=9.9, S D=0.6$, age range $=9.0-10.8$ years, 13 females $)$, 28 older children $(\mathrm{OC}$, mean age $=12.0, S D=0.6$, age range $=11.0-12.8$ years, 14 females), 31 younger adults 
$($ YA, mean age $=22.7, S D=1.6$, age range $=18.8-25.1$ years, 14 females), and 28 older adults $(\mathrm{OA}$, mean age $=$ $67.8, S D=3.0$, age range $=63.9-74.5$ years, 14 females) . Participants of all ages including children were able to sustain their attention for the entire duration of the experiment.

\section{Psychological and audiological assessment}

Psychological and auditory assessments were carried out on a different day before the study. The cognitive battery of the Berlin Aging Study (BASE; Baltes \& Mayer, 1999) was used for psychological assessment. The Identical Pictures (IP) test from this battery - a marker test of perceptual speed - was selected for correlational analysis of relations with electrophysiological data. The materials and procedural details of this test have been described elsewhere (Lindenberger, Mayr \& Kliegl, 1993). In the IP test, a total of 32 items is presented. For each item, a target figure is presented in the upper half of the screen, and five response alternatives are presented in the lower half. Participants have to touch the correct (identical) figure in the lower half as fast as possible. Before the test phase, instructions and three practice items were given. Testing was terminated automatically after $80 \mathrm{~s}$. The score refers to the number of correct responses.

For auditory assessment, the standard HughsonWestlake procedure for measuring pure-tone detection thresholds (0.25-8.0 kHz, bilaterally) was used (Carhart \& Jerger, 1959). Thresholds obtained for left and right sites were averaged, and a two-way repeated measures ANOVA with a between-subjects factor Age and a within-subject factor Frequency $(4 \times 8)$ was carried out. Fischer's LSD test was used for post-hoc testing of age group differences. Statistical analysis revealed a significant main effect of the factors Age, $F(3,107)=$ $47.0, p<.01$, and Frequency, $F(7,749)=18.1, p<.01$, as well as a significant Age by Frequency interaction, $F(21,749)=30.1, p<.01$. Fischer's LSD post-hoc tests revealed significant differences between $\mathrm{OA}$ and other age groups (all $p \mathrm{~s}<.01$ ). Auditory thresholds were highest in OA and generally lowest in YA (see Table 1 for details). For OA, thresholds were particularly elevated at high frequencies. Age differences were least pronounced in the frequency range of stimuli used for the oddball task $(800-1000 \mathrm{~Hz})$. However, statistical analysis of age differences in this frequency range (e.g. $1000 \mathrm{~Hz}$ ) showed significant differences between $\mathrm{OA}$ and other age groups (for all age group comparisons, $p<.05$ ).

\section{Procedure}

The EEG measurement began with a 3-minute relaxation phase (1.5 minutes with eyes closed and 1.5 minutes with eyes open). Relaxation served to adapt the participants to the lab environment and was followed by the auditory oddball task. During the task, participants sat in a chair in a relaxed position in an electrically shielded room,
Table 1 Means and standard deviations for the auditory thresholds (dBSPL) at the different frequency levels between 250 and $8000 \mathrm{~Hz}$ in the four age groups

\begin{tabular}{lcrrr}
\hline & YC $(n=24)$ & OC $(n=28)$ & YA $(n=31)$ & OA $(n=28)$ \\
\hline $250 \mathrm{~Hz}$ & $15.4(6.4)$ & $14.7(6.7)$ & $9.9(6.4)$ & $18.6(12.7)$ \\
$500 \mathrm{~Hz}$ & $14.0(5.3)$ & $11.7(5.3)$ & $9.2(5.7)$ & $17.9(11.5)$ \\
$1000 \mathrm{~Hz}$ & $15.6(5.3)$ & $15.4(6.2)$ & $13.6(4.2)$ & $21.4(11.2)$ \\
$2000 \mathrm{~Hz}$ & $14.0(4.6)$ & $14.2(4.8)$ & $11.5(5.1)$ & $25.1(13.6)$ \\
$3000 \mathrm{~Hz}$ & $11.6(4.1)$ & $11.0(5.4)$ & $8.8(6.7)$ & $30.3(17.3)$ \\
$4000 \mathrm{~Hz}$ & $10.0(4.8)$ & $9.5(4.8)$ & $6.9(6.9)$ & $38.0(17.9)$ \\
$6000 \mathrm{~Hz}$ & $8.1(5.4)$ & $8.5(7.0)$ & $5.1(7.1)$ & $37.8(17.1)$ \\
$8000 \mathrm{~Hz}$ & $12.9(7.3)$ & $15.0(9.0)$ & $8.8(5.7)$ & $50.6(20.3)$ \\
\hline
\end{tabular}

Note: $\mathrm{YC}=$ younger children, $\mathrm{OC}=$ older children, $\mathrm{YA}=$ younger adults, $\mathrm{OA}=$ older adults.

with their eyes closed. They heard two different tone pips: a frequent $1000 \mathrm{~Hz}$ tone as a standard stimulus and a rare $800 \mathrm{~Hz}$ tone as a deviant stimulus. The standard and deviant stimuli were presented binaurally (with a probability of 0.8 and 0.2 for standard and deviant, respectively) through headphones (Sony DJ MDR-V300) at $70 \mathrm{~dB}$ SPL with a duration of $70 \mathrm{~ms}$ (including 10-ms rise and fall time). Stimuli were generated with the software Audacity 1.2.4. The inter-stimulus interval ranged from 1200 to $1500 \mathrm{~ms}$. There were two different experimental conditions: passive listening (unattended) and active counting (attended). In the first condition, participants merely listened to the tone pips without any response, whereas in the second condition, they had to attend to stimuli and to count the deviant tones. After the session, they were asked to report their counting results. Each experimental condition contained 152 standard tones and 38 deviant tones presented in a pseudo-random order fixed for all participants. The order of the conditions was always the same, with the active counting condition following the passive listening condition.

\section{EEG recordings and analyses}

The electroencephalogram (EEG) was recorded from 58 $\mathrm{Ag} / \mathrm{AgCl}$ electrodes using an elastic cap (Electrocap International), with a sampling rate of $500 \mathrm{~Hz}$ in a frequency band ranging from 0.5 to $100 \mathrm{~Hz}$. The left mastoid was used as a reference and the right mastoid was recorded as an active channel. The electrodes were placed according to the international 10-10 system. Vertical and horizontal electrooculogram (EOG) was recorded for control of eye blinks and eye movements. The EEG recordings were corrected for eye movements using the Gratton and Coles algorithm (Gratton, Coles \& Donchin, 1983), and blink artefacts were rejected based on the gradient criterion, that is, a maximum admissible voltage step $(50 \mu \mathrm{V})$, and a difference criterion, that is, a maximum admissible absolute difference between two values in a segment $(200 \mu \mathrm{V})$.

Using a complex Gabor expansion function, EEG time series $(500 \mathrm{~ms}$ pre-stimulus and $1000 \mathrm{~ms}$ after 
stimulus onset) were transformed into a complex timefrequency signal $y\left(f_{n}, t\right)$ for frequencies up to $20 \mathrm{~Hz}$ with a frequency resolution of $0.66667 \mathrm{~Hz}$. Four different measures were obtained from these complex timefrequency matrixes: (i) evoked power, which was calculated from individual ERPs according to $E P\left(f_{n}, t\right)=\left|y\left(f_{n}, t\right)\right|^{2}$, for each frequency bin $f_{n}$ and time lag $t$, (ii) whole power, calculated as the average of the power of a signal in single trials: $W P\left(f_{n}, t\right)=\operatorname{Avg}\left(\left|y_{i}\left(\mathrm{f}_{\mathrm{n}}, t\right)\right|^{2}\right)$, where $i$ indicates number of single trials, (iii) phase locking index (PLI) defined by $\operatorname{PLI}\left(f_{n}, t\right)=\left|\left\langle e^{j \cdot \Phi^{k}\left(f_{n}, t\right)}\right\rangle\right|, j=\sqrt{-1}$, and phase coherence (PC) defined by $P C_{\Phi}\left(f_{n}, t\right)=\left|\left\langle e^{j \cdot \Delta \Phi^{k}\left(f_{n}, t\right)}\right\rangle\right|, j=\sqrt{-1}$, where phase difference between two electrodes $\left(\Delta \Phi^{k}=\right.$ $\left.\bmod \left(\Phi_{1}^{k} f_{n}, t\right)-\bmod \left(\Phi_{2}^{k} f_{n}, t\right), 2 \cdot \pi\right)$, with instantaneous phases of two electrodes across k trials $\left(\Phi_{1}^{k} f_{n}, t\right)=\arg \left\{y_{1}^{k}\left(f_{n}, t\right)\right\}$ and $\left(\Phi_{1}^{k} f_{n}, t\right)=\arg \left\{y_{2}^{k}\left(f_{n}, t\right)\right\}$. For time-frequency presentations and statistical analyses, the average PC of four midline electrodes relative to 16 other electrodes of the 10-20 system was computed (for example, $F z$ to Fpz, F7, F3, F4, . . , Oz; $C z$ to Fpz, F7, F3, Fz, F4, . ., Oz, etc.). All analyses were carried out with an equal number of trials for deviant and standard stimuli. To minimize sequence effects, standard stimuli were always chosen in the middle of two deviants, i.e. the distances between standard and deviant stimuli were equally distributed.

These four measures subdivided into three equidistant frequency bands from 0 to $12 \mathrm{~Hz}$ (delta, theta and alpha) and averaged for the pre-stimulus interval between -350 and $-150 \mathrm{~ms}$ and five consecutive 200-ms post-stimulus time intervals, were statistically analyzed using a five-way repeated measures ANOVA with a between-subjects factor Age (four age groups: YC, OC, YA, and $\mathrm{OA}$ ) and four within-subject factors: Task (attended vs. unattended), Stimulus (deviant vs. standard), Site (four different electrodes: $\mathrm{Fz}, \mathrm{Cz}, \mathrm{Pz}$ and $\mathrm{Oz}$ ), and Time (6 time intervals). In all ANOVAs, GreenhouseGeisser epsilons were used for non-sphericity correction when necessary. Fischer's LSD test was employed for post-hoc testing of age group differences.

To assess correlations between cognitive performance and electrophysiological data, Pearson product correlations were computed separately for each age group between the scores in the IP task (raw scores) and the synchronization measures. Analyses were restricted to trials with a deviant stimulus under attended and unattended conditions at four electrode sites. Five consecutive 200-ms time intervals after stimulus onset (T1-T5) were considered. In accordance with our hypotheses that the relation between EEG measures and IP scores should be strongest at synchronization maxima, 40 predictors (10 for each of four electrodes), that is, regions of interest (ROI) showing high synchronization patterns, were chosen for correlational analyses. EEG measures showing strong correlations with IP scores were submitted to multiple regression analyses to assess the predictive value of age and EEG indicators
Table 2 Means and standard deviations for the four spectral power and synchronization measures (PLI, EP, WP, and PC) in the four age groups for delta, theta, and alpha frequency bands

\begin{tabular}{ccccc}
\hline & YC & OC & YA & OA \\
\hline PLI & & & & \\
Delta & $0.23(0.05)$ & $0.28(0.06)$ & $0.35(0.09)$ & $0.37(0.09)$ \\
Theta & $0.20(0.03)$ & $0.21(0.03)$ & $0.24(0.05)$ & $0.25(0.04)$ \\
Alpha & $0.17(0.02)$ & $0.17(0.03)$ & $0.18(0.03)$ & $0.19(0.04)$ \\
EP & & & & \\
Delta & $1.13(0.32)$ & $1.25(0.35)$ & $1.04(0.38)$ & $0.79(0.26)$ \\
Theta & $0.58(0.15)$ & $0.57(0.20)$ & $0.44(0.15)$ & $0.32(0.08)$ \\
Alpha & $0.37(0.10)$ & $0.40(0.16)$ & $0.36(0.14)$ & $0.23(0.07)$ \\
WP & & & & \\
Delta & $3.77(0.62)$ & $3.53(0.61)$ & $2.27(0.54)$ & $1.68(0.52)$ \\
Theta & $2.30(0.48)$ & $2.12(0.62)$ & $1.44(0.44)$ & $0.99(0.28)$ \\
Alpha & $1.76(0.43)$ & $2.03(0.75)$ & $1.68(0.67)$ & $0.99(0.35)$ \\
PC & & & & \\
Delta & $0.47(0.05)$ & $0.51(0.06)$ & $0.62(0.06)$ & $0.65(0.08)$ \\
Theta & $0.45(0.06)$ & $0.48(0.07)$ & $0.56(0.05)$ & $0.61(0.08)$ \\
Alpha & $0.42(0.06)$ & $0.47(0.09)$ & $0.53(0.05)$ & $0.58(0.06)$ \\
\hline
\end{tabular}

Note: $\mathrm{YC}=$ younger children, $\mathrm{OC}=$ older children, $\mathrm{YA}=$ younger adults, $\mathrm{OA}=$ older adults; PLI $=$ phase locking index, $\mathrm{EP}=$ evoked power, $\mathrm{WP}=$ whole power, $\mathrm{PC}=$ phase coherence.

and their interaction on the IP performance. Correlations were transformed into Fisher's $z$-scores to test whether they were reliably different from each other.

\section{Results}

To provide an overview of the observed lifespan differences, Table 2 reports averages of the four midline electrodes across the six time intervals for the four spectral power and synchronization measures for deviant stimuli under attended conditions.

\section{Lifespan differences in phase synchronization of neural oscillations across trials}

We used PLI to determine phase synchronization across the trials (see Methods) grouped into standard and deviant stimuli during passive listening (unattended) and active counting (attended) conditions at the four midline electrode positions ( $\mathrm{Fz}, \mathrm{Cz}, \mathrm{Pz}$ and $\mathrm{Oz}$ ). Grand averages of PLI and corresponding ERPs (event-related brain potentials) across subjects in the four age groups are shown for a representative central electrode site $(\mathrm{Cz})$ in Figure 1 (regarding ERPs see Mueller, Brehmer, von Oertzen, Li \& Lindenberger, 2008). ERPs were calculated using a $50-\mathrm{ms}$ pre-stimulus baseline correction and band pass Butterworth zero-phase digital filtering $(0.5-20 \mathrm{~Hz})$. Phase synchronization increased with advancing age across all experimental conditions, demonstrating the global character of this phenomenon.

A five-way repeated measures ANOVA (Age $\times$ Task $\times$ Stimulus $\times$ Site $\times$ Time) revealed a significant main effect of the factor Age for delta, $F(3,107)=25.9, p<.01$, and theta, $F(3,107)=9.9, p<.01$, frequencies, with significant differences between children and adults determined 


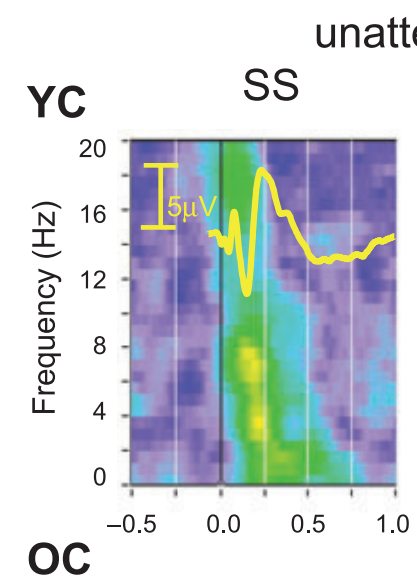

unattended

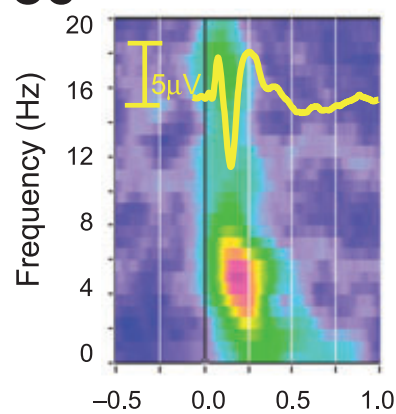

YA

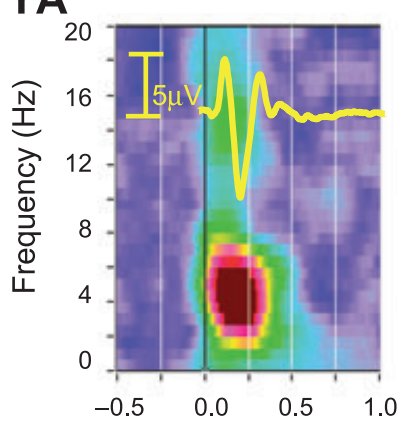

OA

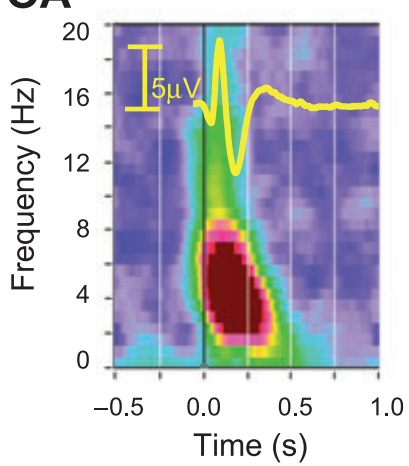

DS
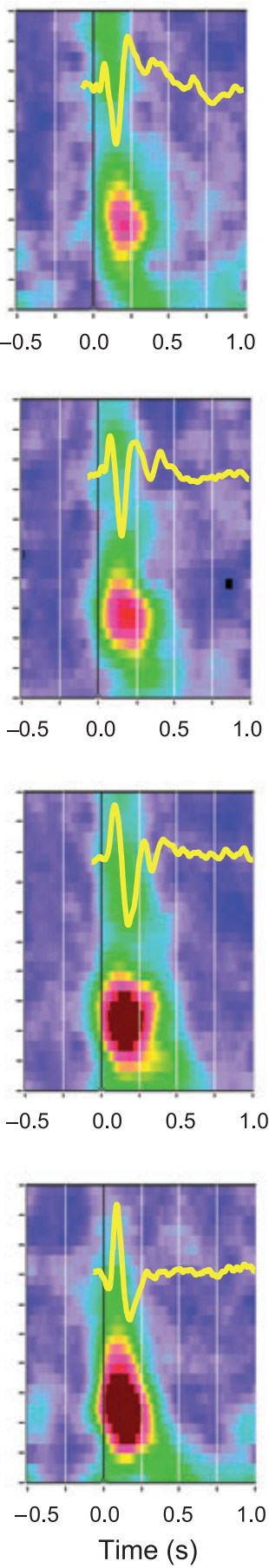

attended

SS
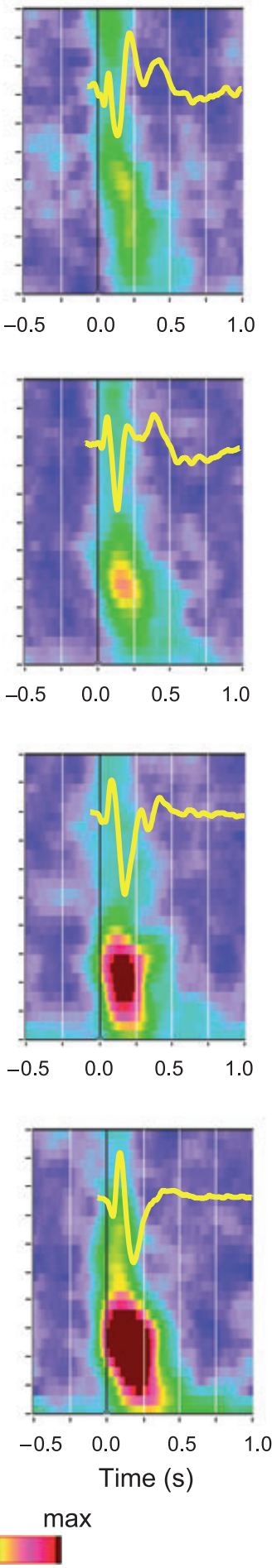
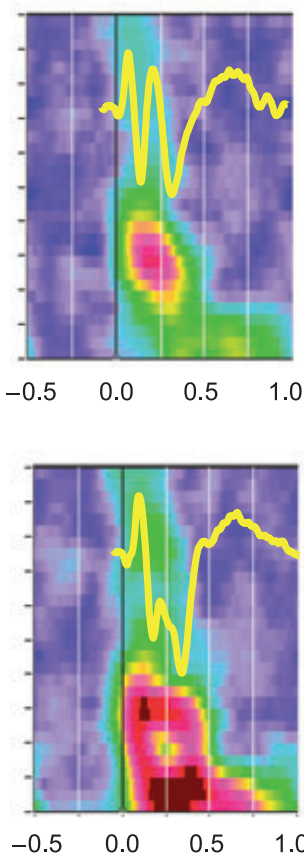

DS
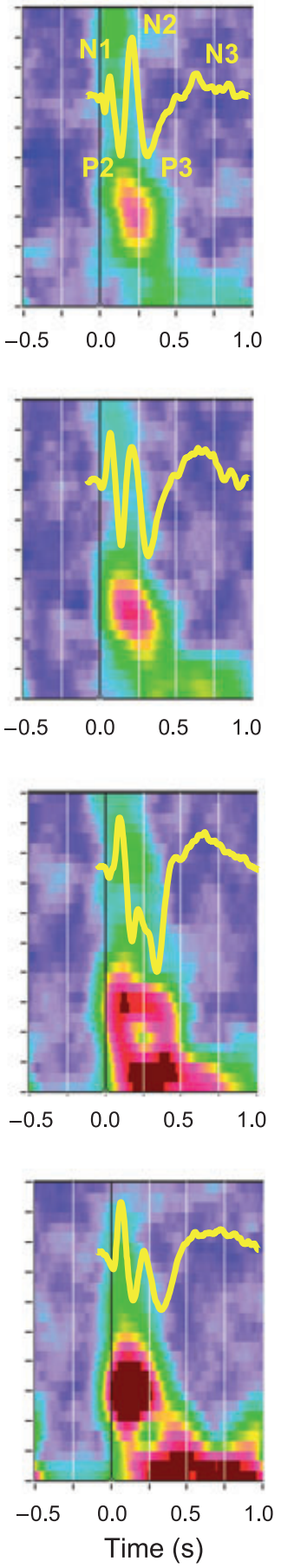

Figure 1 Grand average stimulus-locked time-frequency maps of phase locking index (PLI) and grand average ERP waveforms (yellow curves) at the Cz electrode for standard (SS) and deviant (DS) stimuli under unattended (left two columns) and attended (right two columns) conditions by age group; (YC) younger children, (OC) older children, (YA) younger adults, and (OA) older adults. Phase locking increases with age, and is more pronounced under the attended deviant condition (right column). Faster ERP components (e.g. N1, P2, N2 peaking at about 100, 150, and $200 \mathrm{~ms}$, respectively) are related to theta/alpha PLI maxima and slower ERP components (e.g. P3, N3 peaking at about 300 and 600 ms, respectively) are related to delta/theta PLI maxima. The latter maximum is most clearly seen under the attended deviant condition (right column) in groups of younger and older adults. Children showed a strong P3/N3 complex accompanied by low levels of phase locking. 
by Fischer's LSD post-hoc tests again for delta (OA, YA $>\mathrm{YC}, \mathrm{OC}$, all $p \mathrm{~s}<.01)$ and theta (YA, OA > YC, OC, all $p \mathrm{~s}<.05)$ frequencies. As shown by the significant interactions, age differences in the delta frequency band were higher under attended than unattended conditions, $F(3,107)=8.3, p<.01$, and higher for deviant than for standard stimuli, $F(3,107)=4.5, p<.01$. These differences were highest fronto-centrally, $F(9,321)=5.6$, $p<.01$, and during the first and the second time interval after stimulus onset, $F(15,535)=9.4, p<.01$. In the theta frequency band, age differences were also higher under attended than unattended conditions, $F(3,107)=$ $3.7, p<.05$, and highest fronto-centrally, $F(9,321)=8.6$, $p<.01$, and during the first time interval after stimulus onset, $F(15,535)=17.0, p<.01$. In the alpha frequency band, the Age by Time interaction was significant, with higher PLI values during the first time interval in OA compared to other age groups, $F(15,535)=3.9, p<$ .01 . In sum, older adults showed globally the highest PLI, indicating strong phase synchronization across trials.

\section{Lifespan differences in evoked and whole power spectra}

Evoked and whole power (Buzsáki, 2005; CastroAlmanancos \& Connors, 1996) were determined with complex Gabor transforms to achieve adequate timefrequency resolution of amplitude estimates reflecting evoked and induced (not phase-locked) activity, correspondingly. Both measures were determined separately for standard and deviant stimuli under passive listening (unattended) and active counting (attended) conditions at four midline electrodes. Grand averages of evoked and whole power across subjects are shown separately for each age group at the $\mathrm{Cz}$ electrode location for the attended deviant condition (Figure 2). In contrast to PLI, evoked power and whole power in particular decreased with advancing age. Relative to children, younger adults showed lower evoked power, and strongly reduced whole power. In other words, in younger adults, the power in single trials was transformed to a greater degree into evoked power through higher phase synchronization than in children.

This was also true for older adults, with the difference that the power in single trials, as expressed by WP, was small to begin with, so that EP - despite high phase synchronization - was lower than in children and even lower than in younger adults. Thus, children showed higher power or energy costs while working on the task, whereas adults showed higher levels of phase synchronization, suggesting that task-relevant cortical mechanisms varied by age.

A statistical analysis of EP and WP revealed a significant main effect of the factor Age for all three frequency bands (EP, delta: $F(3,107)=36.9$; EP, theta: $F(3,107)=$ 22.5, EP, alpha: $F(3,107)=13.6$; WP, delta: $F(3,107)$ 99.9; WP, theta: $F(3,107)=34.9$, WP, alpha: $F(3,107)$
YC

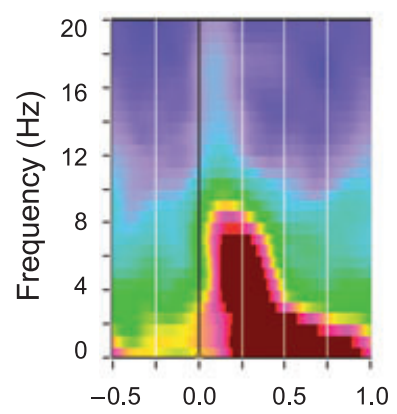

OC

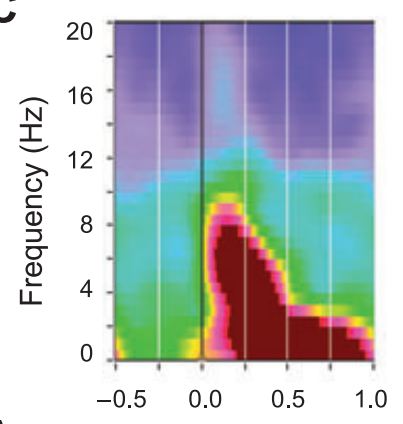

YA

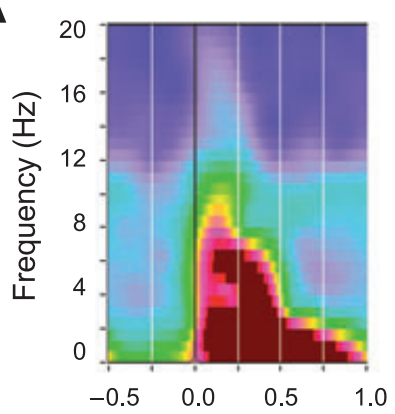

OA

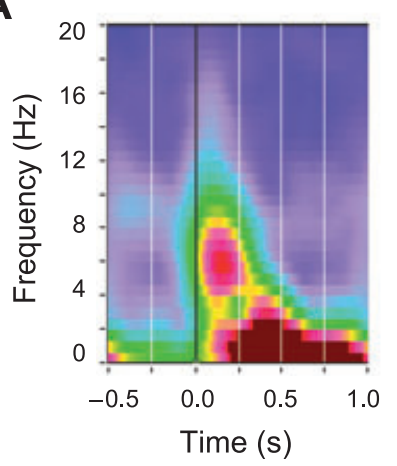

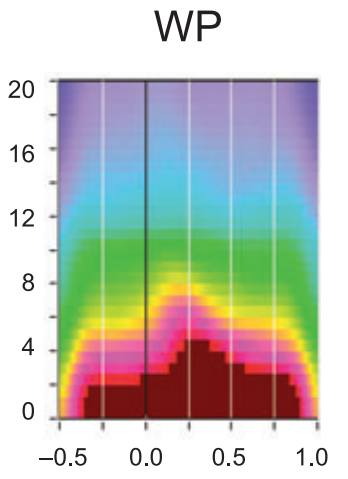
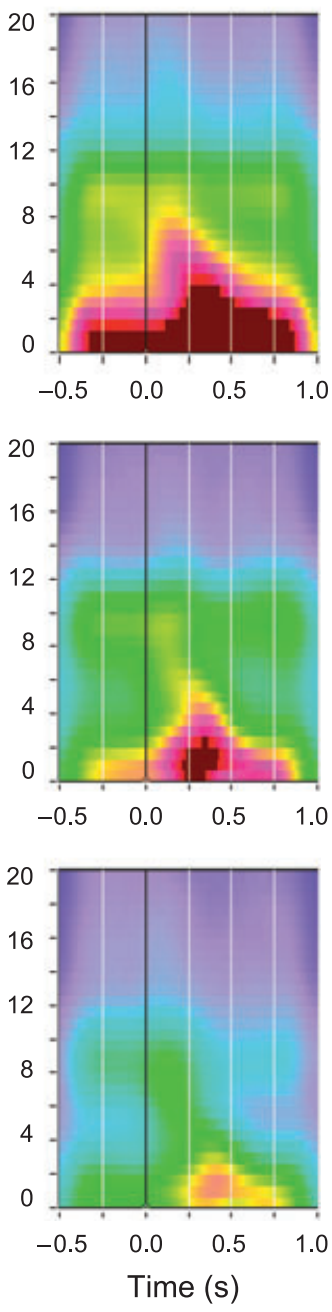

$\max$
Figure 2 Grand average time-frequency maps of evoked power (EP) and whole power (WP) at the Cz electrode for deviant stimuli under the attended condition by age group; (YC) younger children, (OC) older children, (YA) younger adults, and (OA) older adults. Note that EP and WP have different scaling, but that the scaling within EP and WP across ages remains invariant. EP and especially WP decrease with advancing age.

$=16.2$; all $p<.01)$. For both EP and WP, Fischer's LSD post-hoc tests showed significant age differences between children and adults as well as between young and older adults $(\mathrm{YC}, \mathrm{OC}>\mathrm{YA}>\mathrm{OA}$, all $p$ s $<.01)$ in the delta and 
theta frequencies. In the alpha frequency, differences between children and younger adults were not significant. There were significant interactions for Age by Task for EP in the delta frequency band, $F(3,107)=3.3, p=$ .023 , Age by Site for both EP (delta: $F(9,321)=2.6$, $p=.020$; theta: $F(9,321)=4.6, p<.01$, and alpha: $F(9,321)=4.7, p<.01)$ and WP (theta: $F(9,321)=3.3$, $p<.01$, and alpha: $F(9,321)=6.9, p<.01)$ in all frequency bands except WP at delta frequency, and Age by Time except EP at alpha frequency (EP, delta: $F(15,355)=4.4$; EP, theta: $F(15,355)=4.4$, EP; WP, delta: $F(15,355)=18.9$; WP, theta: $F(15,355)=11.3$, WP, alpha: $F(15,355)=7.6$; all $p \mathrm{~s}<.01)$. Taken together, these interactions indicate that the age differences in EP and WP also depended on other factors, but generally decreased with advancing age.

\section{Lifespan differences in phase synchronization of neural} oscillations between brain sites

In addition to PLI and power measures, we also determined phase synchronization or phase coherence (PC) between different electrode locations to identify differences in synchronization between distinct brain areas. More detailed spatiotemporal information is provided by phase synchrony over the scalp (Figure 3). For this purpose, PC was averaged within two overlapping 400-ms time intervals for delta (200-600 ms) and theta (0-400 ms) frequencies. For statistical analysis, we then calculated the average $\mathrm{PC}$ for the four midline electrodes to 16 other electrodes distributed over the scalp (see Methods). Grand averages of PC, as shown in Figure 3 for the $\mathrm{Cz}$ electrode, again indicate a continuous increase in synchronization with advancing age. This increase was not local in character but distributed over the entire scalp (Figure 3). Here, we refrain from interpreting age differences in PC between specific pairs of electrodes and restrict our analyses to average age trends in PC, representing age differences in synchronization patterns at a global (unspecific) level. Like other measures, average PC was determined for the 200-ms pre-stimulus and five consecutive 200-ms post-stimulus time intervals and analyzed with five-way repeated measures ANOVA (Age $\times$ Task $\times$ Stimulus $\times$ Site $\times$ Time) separately for each frequency band.

A statistical analysis of the average PC revealed a significant main effect of the factor Age for all three frequency bands (delta: $F(3,107)=35.2$; theta: $F(3$, $107)=30.1$, and alpha: $F(3,107)=19.4$; all $p s<.01)$. Fischer's LSD post-hoc tests showed significant age differences between children and adults for all three frequency bands, significantly higher $\mathrm{PC}$ in OC than in $\mathrm{YC}$ at the delta and alpha frequency $(\mathrm{OA}, \mathrm{YA}>\mathrm{OC}>$ $\mathrm{YC}$, all $p \mathrm{~s}<.05$ ) and significantly higher $\mathrm{PC}$ in OA than $\mathrm{YA}$ at the theta frequency $(\mathrm{OA}>\mathrm{YA}>\mathrm{YC}$, OC, all $p \mathrm{~s}<.01)$. Significant interactions Age $\times$ Task (delta: $F(3,107)=2.8, p=.045$; theta: $F(3,107)=4.2, p<.01$, and alpha: $F(3,107)=3.2, p=.026)$ and Age $\times$ Time (delta:
$F(15,535)=3.8, p<.01$; theta: $F(15,535)=1.8, p=$ .038 , and alpha: $F(15,535)=1.8, p=.021)$ for all three frequency bands, as well as Age $\times$ Site (at the delta: $F(9$, $321)=2.3, p=.039$ and theta frequency: $F(3,107)=5.2$, $p<.01$ ) and Age $\mathrm{x}$ Stimulus (at the alpha frequency: $F(3,107)=5.1, p<.01)$ suggest that the age-specific differences were greater under attended than unattended task conditions, greater for deviant than for standard stimuli, as well as greater in the middle time interval and at central electrode location.

\section{Correlations between synchronization and independently assessed measures of perceptual speed}

To assess the functional status of the synchronization measures, we correlated them with scores obtained in the Identical Pictures (IP) test, an index of perceptual speed. Children generally showed non-significant correlations, with no systematic relations to synchronization maxima; exceptions were some positive correlations in $\mathrm{OC}$ under both attended and unattended conditions (for attended conditions at the delta frequency: PLI/Fz/T2: $R=0.45$, $p=.017 ; \mathrm{EP} / \mathrm{Oz} / \mathrm{T} 2: R=0.50, p=.0064 ; \mathrm{EP} / \mathrm{Oz} / \mathrm{T} 5$ : $R=0.45, p=.017 ; \mathrm{PC} / \mathrm{Fz} / \mathrm{T} 1: R=0.41, p=.030$; $\mathrm{PC} / \mathrm{Fz} / \mathrm{T} 2: R=0.42, p=.026 ; \mathrm{PC} / \mathrm{Oz} / \mathrm{T} 1: R=0.37, p$ $=.050 ; \mathrm{PC} / \mathrm{Oz} / \mathrm{T} 2: R=0.39, p=.040$; for unattended conditions at the alpha frequency: EP/Pz/T1: $R=0.39$, $p=.038$ ). In contrast, younger adults showed positive correlations of substantial magnitude for PLI and EP in the delta and theta frequency across all electrode locations under attended conditions, and older adults showed negative correlations for PLI and EP in the theta and alpha frequency, also across almost all electrode sites under attended conditions (see Table 3). The significant correlations among OA were found primarily for the first time interval after stimulus onset. In contrast, correlations in YA appeared up to $200 \mathrm{~ms}$ later (see Figures 4 and 5 for PLI and EP, respectively).

Multiple regression analyses with the predictor variables Age (YA vs. OA), EEG Indicator, and the interaction term Age $\times$ EEG Indicator showed significant multiple correlations in all cases for both PLI $\left(R^{2}=0.67-0.74\right.$, $F(3,55)=36.6-53.4$, all $p$ s $<.01)$ and $\mathrm{EP}\left(R^{2}=0.66\right.$ $0.72, F(3,55)=36.6-48.1$, all $p$ s $<.01)$. The interaction term Age $\times$ EEG Indicator was significant for PLI at delta and theta frequency bands, especially centrally and parietally, and for EP at theta and alpha frequency bands for the first time interval after stimulus onset (see Table 3 for details). The significant interaction term indicates that PLI and EP predict IP performance differentially in the different age groups, with higher correlations in YA than OA. Accordingly, differences in correlations between YA and OA were statistically significant (Table 3).

Under unattended conditions, correlations were considerably reduced and were restricted in younger adults to $\mathrm{EP}$ in the delta and theta frequencies (delta: EP/Oz/ $\mathrm{T} 3: R=0.42, p=.019$; theta: $\mathrm{EP} / \mathrm{Fz} / \mathrm{T} 2: R=0.42, p=.018$; 
YC
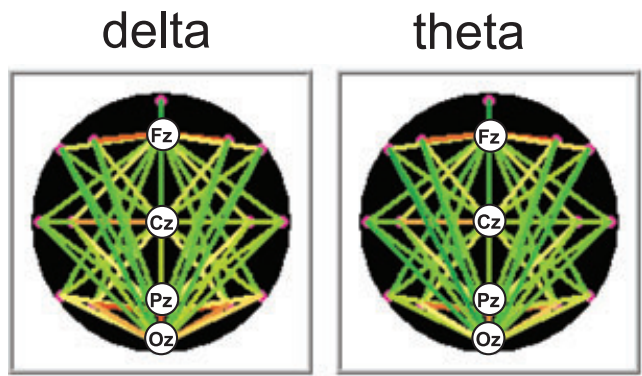

OC
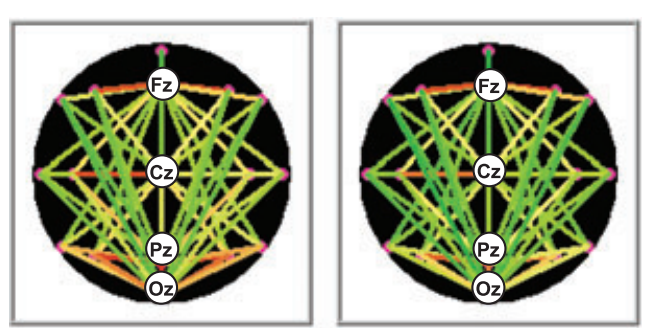

YA
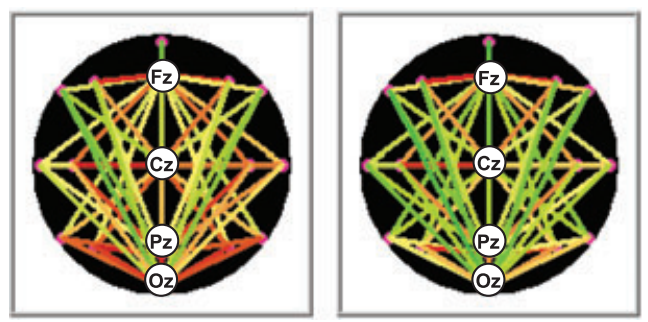

OA
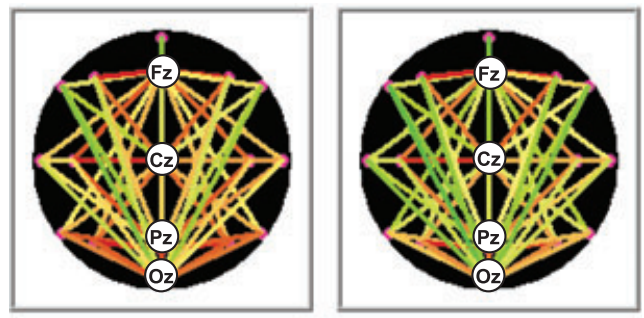

$\min$

$\max$
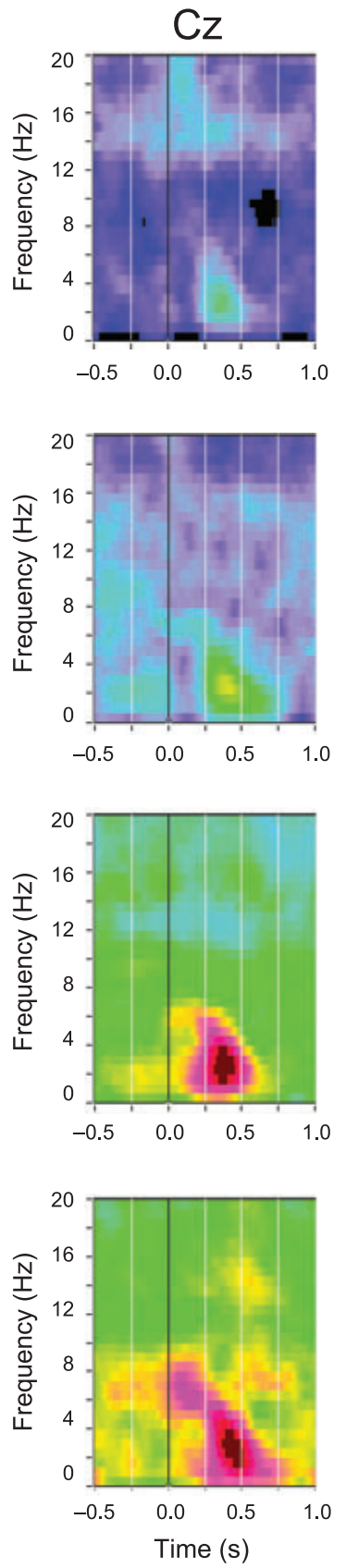

$\min$ $\max$

Figure 3 Lifespan differences in phase coherence. Grand average of phase coherence brain maps for delta and theta frequencies and time-frequency maps of average phase coherence at the Cz electrode for deviant stimuli under the attended condition by age group; (YC) younger children, (OC) older children, (YA) younger adults, and (OA) older adults. In the brain maps, connections between the four midline electrodes and all other electrodes (Fpz, F7, F3, F4, . . , OZ) are represented for the delta frequency averaged over the time interval of 0-400 ms, and for the theta frequency averaged over the time interval of 200-600 ms after stimulus onset. For time-frequency maps, the PC of the $C z$ electrode relative to 16 other electrodes was averaged. Similar to phaselocking (PLI; cf. Figure 1), PC increases with advancing age. This increase applies both to short-range and long-range phase coherence.

$\mathrm{EP} / \mathrm{Fz} / \mathrm{T} 3: R=0.42, p=.019 ; \mathrm{EP} / \mathrm{Cz} / \mathrm{T} 2: R=0.39, p=$ $.032 ; \mathrm{EP} / \mathrm{Fz} / \mathrm{T} 3: R=0.36, p=.048 ; \mathrm{EP} / \mathrm{Pz} / \mathrm{T} 2: R=0.38$, $p=.033)$. Older adults under unattended conditions showed no reliable correlations.

\section{Discussion}

We examined age-related changes in the neural coding of sensory events during auditory perception by means of 
Table 3 Correlations between synchronization measures (PLI and EP) at the different regions of interest (ROI) and IP sCores in young $(\mathrm{n}=31)$ and older $(\mathrm{n}=28)$ adults as well as $t$-values for the interaction term (Age $\times$ EEG Indicator) and $z$-values for correlation differences between the age groups

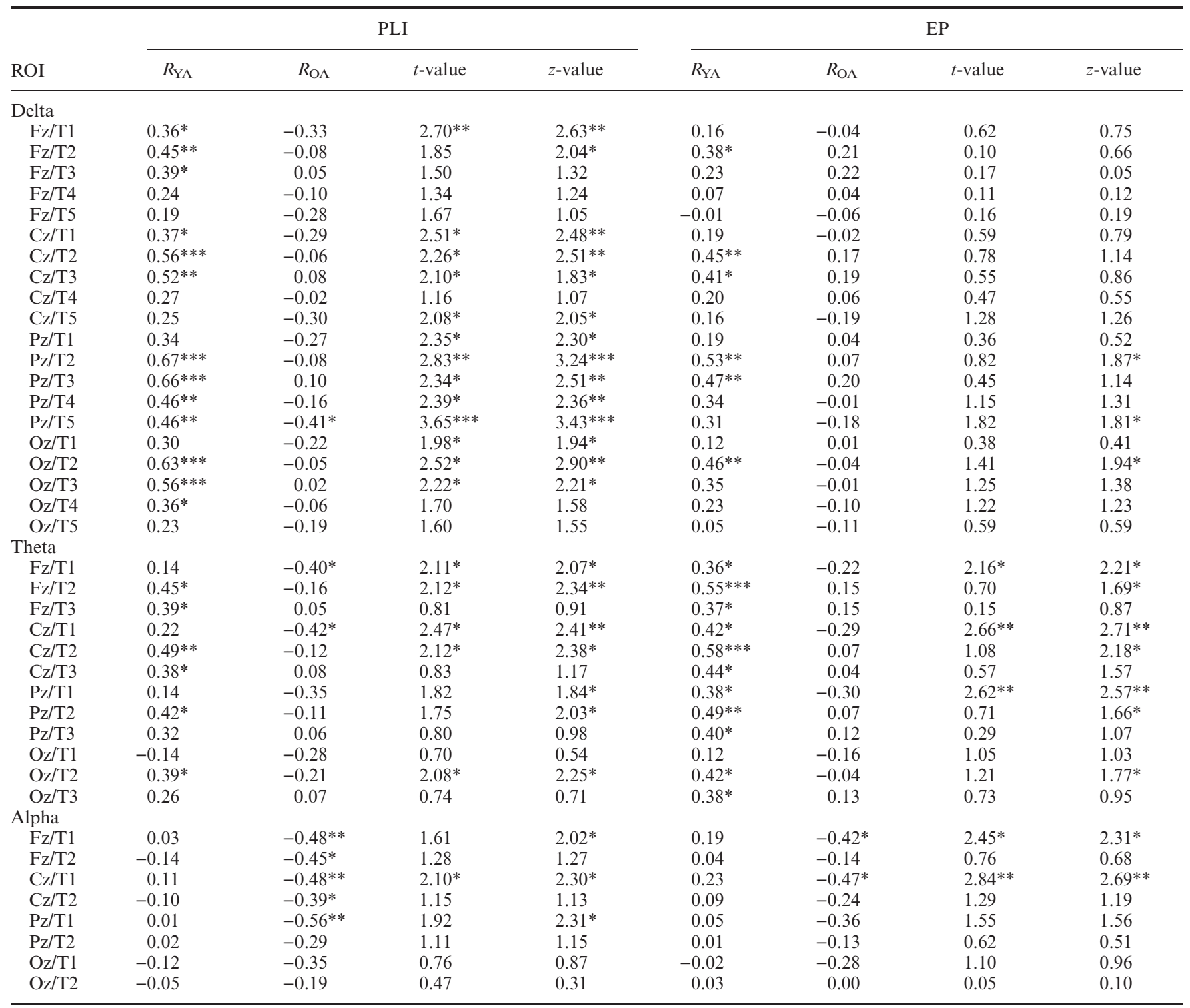

Note: $* p<.05 ; * * p<.01 ; * * * p<.001$.

phase synchronization and spectral power measures during attended and unattended conditions in the auditory oddball task. The main findings are: (a) that phase synchronization increases from childhood to old age, (b) that evoked and especially whole power decrease from childhood to old age. These two age trends are moderated by task condition (higher under attended than unattended conditions) and stimulus type (higher for deviant than standard stimuli) manipulations. In addition, age-related differences also varied by processing stage, frequency domain, and electrode site.

Different mechanisms appeared to be operating in children and adults (especially YC and OA) while working on the task. The increase in phase synchronization with age implies that the overall time structure of neural responses varied considerably across trials in children but was highly stable in both young and older adults. In addition, changes in PC indicate that children showed variable time structures at different electrode locations and weaker phase-related coupling between electrodes, whereas adults (especially older adults) displayed more stable time structures as well as more stable relations between different electrodes, pointing to a high degree of phase coupling between electrode sites. Conversely, children showed higher EEG power than adults, and this may have allowed them to perform the task proficiently despite low degrees of synchronization. Children showed surprisingly high values for whole power across all processing stages and stimulus types. As a consequence, they also showed higher levels of evoked power than adults despite lower levels of phase synchronization. 

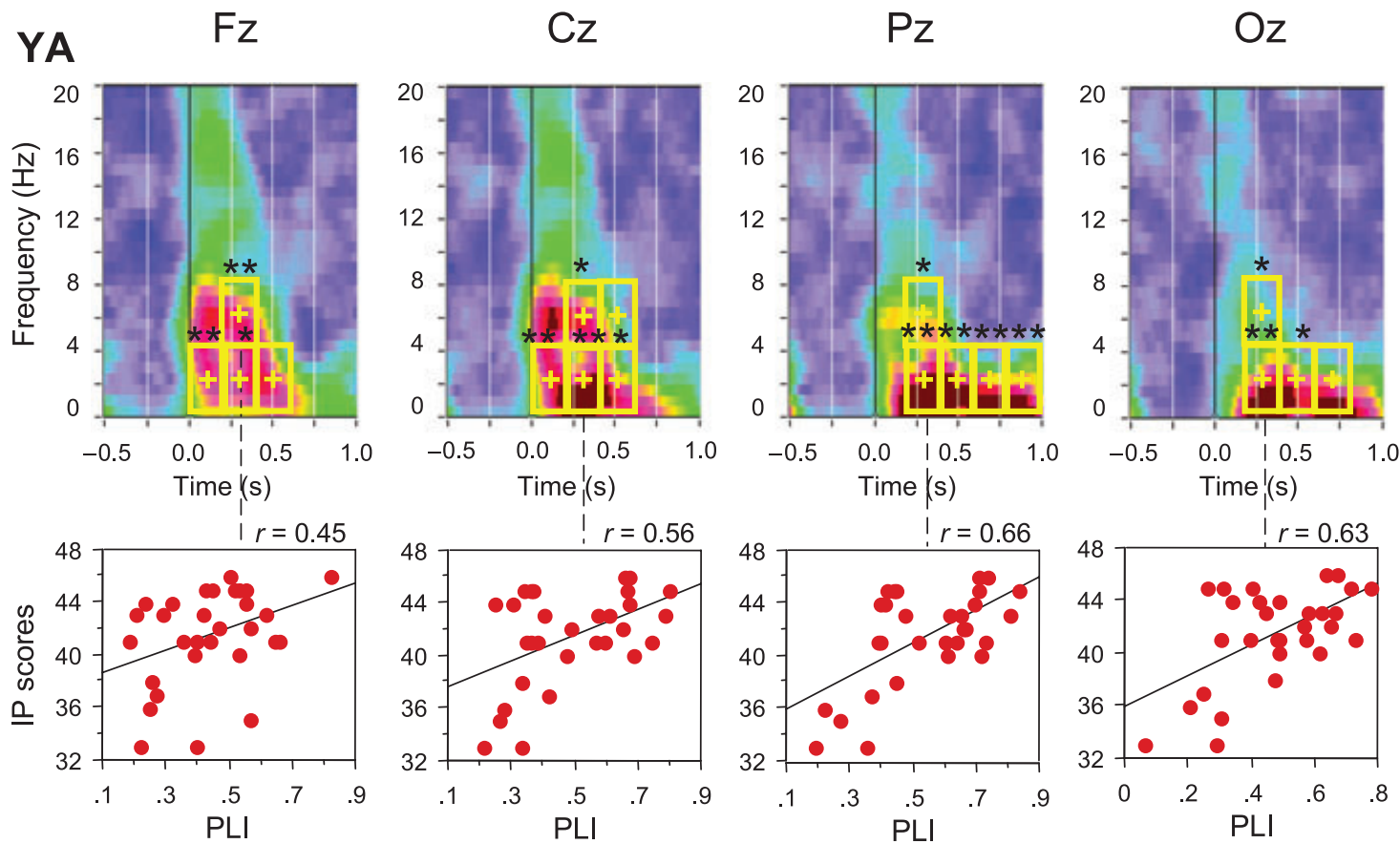

\section{OA}
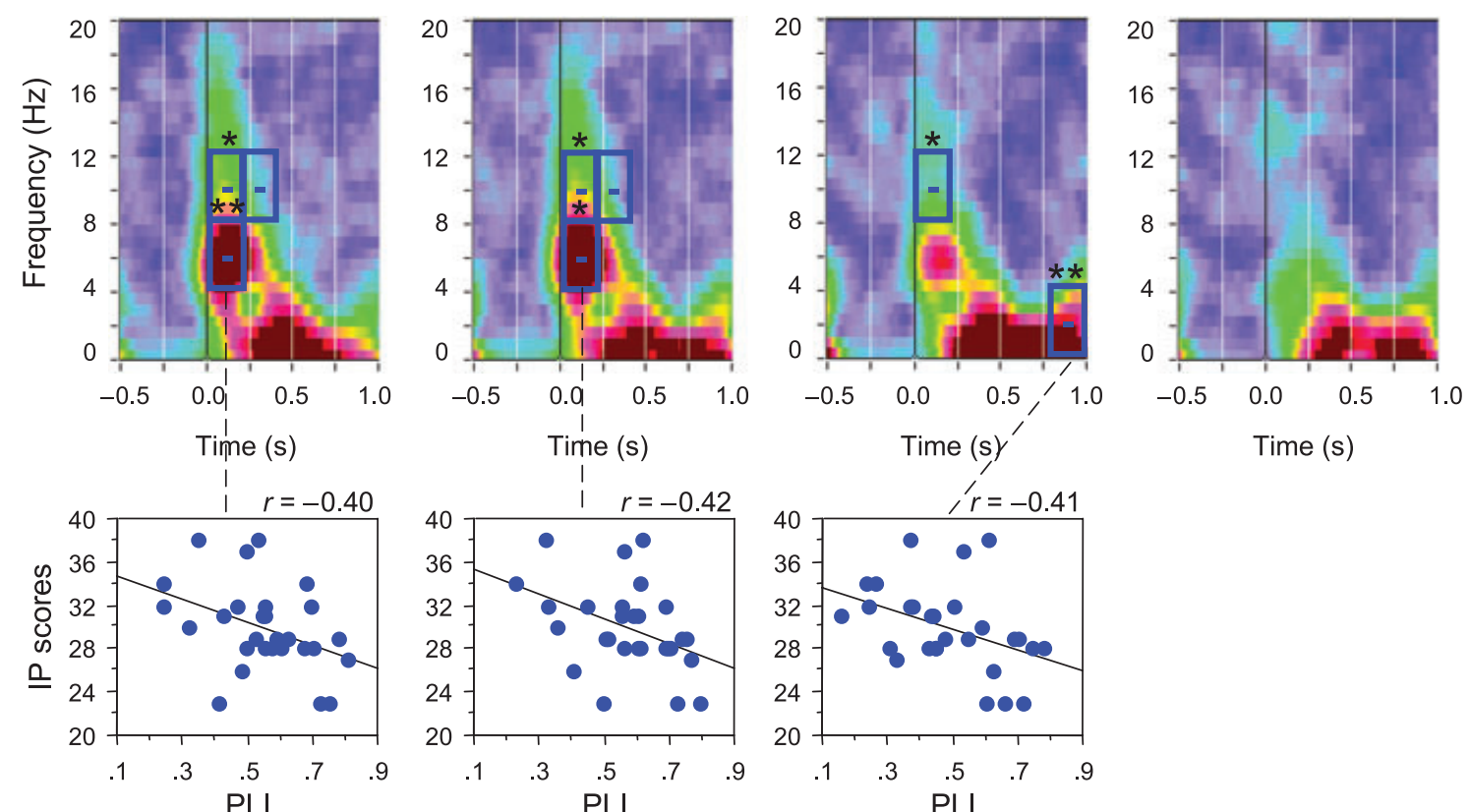

Figure 4 Grand average stimulus-locked time-frequency maps of PLI at the four midline electrode locations $(F z, C z, P z$, and $O z)$ for deviant stimuli under the attended condition and correlation plots between the PLI and IP scores in young (YA) and older $(O A)$ adults. The rectangles mark the time-frequency ranges used to compute average PLI values for statistical analysis (see Methods). Yellow rectangles with a plus sign indicate significant positive correlations, and blue rectangles with a minus sign indicate significant negative correlations. Significant correlations between PLI and IP scores were always positive among younger adults and always negative among older adults. Significant differences in correlation coefficients between $Y A$ and OA are labeled by asterisks:

$* p<.05 ; * * p<.01$.

We can only speculate about the reasons for the observed differences in phase locking between children and adults. Two plausible alternatives come to mind. First, neural activity may have been equally aligned to the stimulus in children and adults (in absolute terms) but may have been buried under a disproportionately large amount of stimulus-independent (background) activity in children, leading to lower phase-locking values (which are relative to the total recorded activity). Second, the phase alignment of neural activity to the stimulus may indeed be more random in children than in adults, on top of differences in total neural activity. 
YA
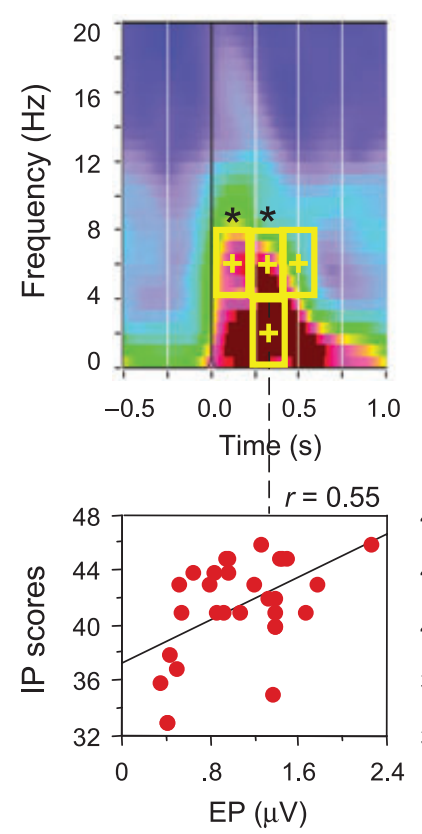

OA

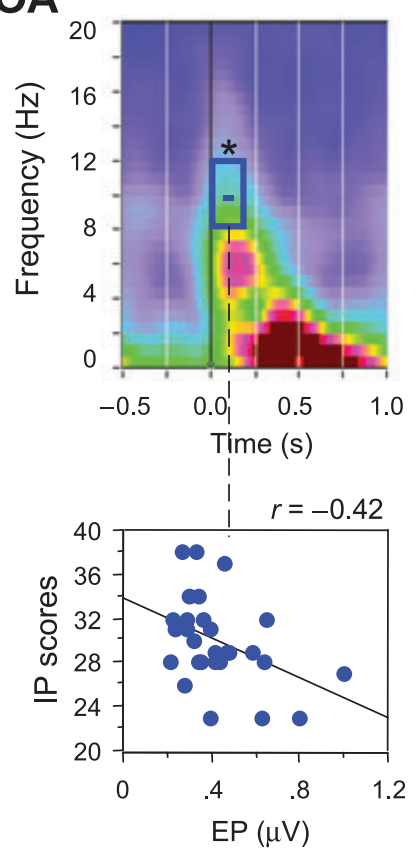

$\mathrm{Cz}$
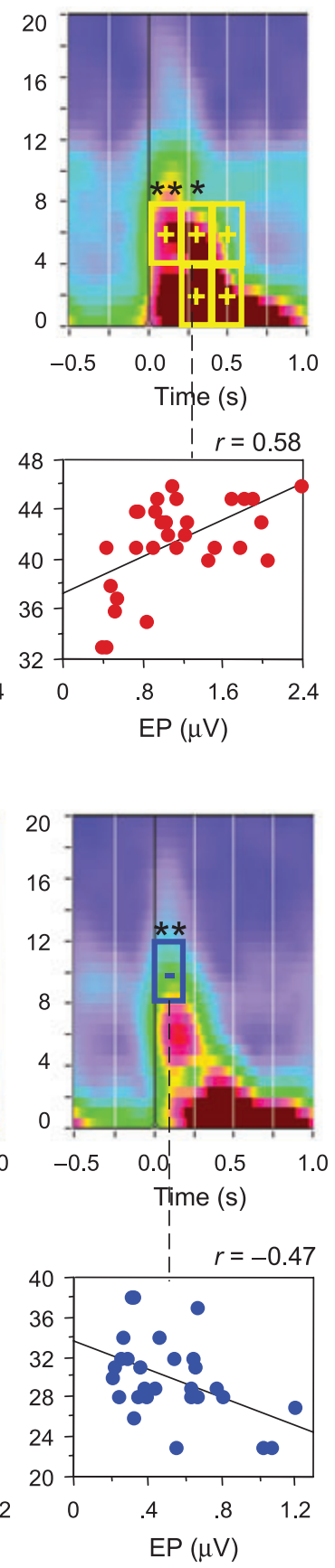

$\mathrm{Pz}$
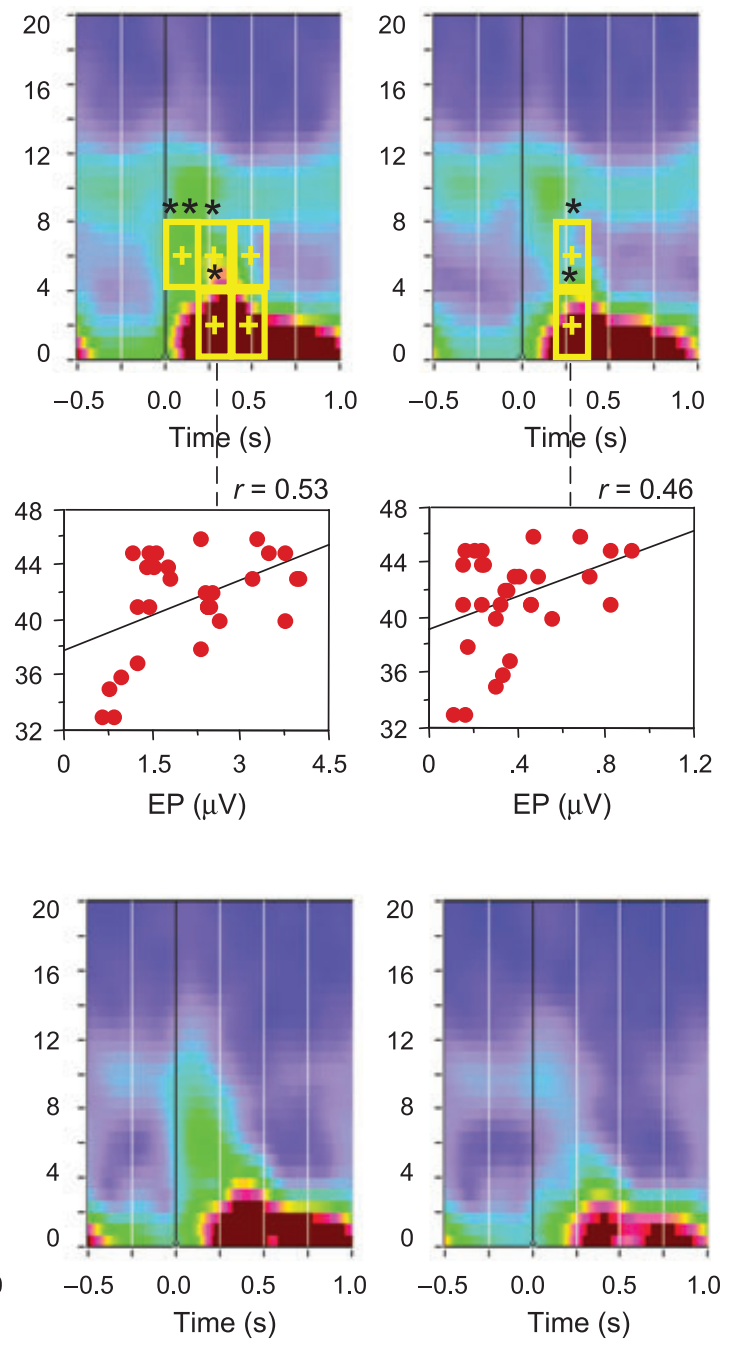

Figure 5 Grand average stimulus-locked time-frequency maps of $E P$ at the four midline electrode locations ( $F z, C z, P z$, and $O z)$ for deviant stimulus under attended condition and correlation plots between the EP and IP scores in young (YA) and older (OA) adults. The rectangles mark the time-frequency ranges used to compute average EP values for statistical analysis. Yellow rectangles with a plus sign indicate significant positive correlations, and blue rectangles with a minus sign indicate significant negative correlations. As with the PLI (see Figure 4), correlations between EP and IP scores were always positive in younger adults and negative in older adults. Significant differences between correlation coefficients in YA and OA are labeled by asterisks: ${ }^{*} p<.05$; ** $p<.01$.

The present data do not permit an unambiguous discrimination between the two alternatives. In either case, it seems likely that the neural code represented by phase alignment was substantially weaker in children than in adults, raising the question whether the processing of children and adults differed in more fundamental ways.
Spectral power measures (i.e. EP and WP) reflect, to a considerable extent, synchronous cell activity under a given electrode (local synchronization). High spectral power means that a substantial number of neural units are simultaneously active (Srinivasan et al., 1999; Varela et al., 2001). Based on this interpretation of spectral 
power, local synchronization was very high in children, but scarcely phase-locked to the stimulus across trials. In contrast, local synchronization was relatively low in adults but strongly phase-locked to the stimulus, as revealed by its oscillatory covariation across trials. Hence, we tentatively propose that the present findings reflect age-related changes in the relative prominence of different mechanisms of neural coding in auditory perception. Adrian and colleagues (Adrian, 1926; Adrian \& Bronk, 1929) showed that the intensity of a stimulus is often coded as the rate of nerve impulses over time. This is commonly referred to as the 'rate code' (Stein, Gossen \& Jones, 2005). According to an alternative coding concept, known as 'temporal coding' or 'synaptic plasticity', the exact timing of neural spikes is also relevant for the coding of sensory and motor events (Hestrin \& Galarreta, 2005; Mehta, Lee \& Wilson, 2002). Synaptic plasticity or Hebbian learning plays a crucial role in information processing including synaptic changes ranging from short-term plasticity or short-term potentiation (STP), involving changes over milliseconds to minutes, to long-term plasticity or long-term potentiation (LTP) with a timescale of hours or longer (Abbott \& Regehr, 2004). Synaptic connections are altered by temporal spatial firing patterns of presynaptic neurons (Abbott \& Regehr, 2004; Destexhe \& Marder, 2004). In other words, the firing strength is dependent on temporal tuning between presynaptic and postsynaptic neurons, suggesting that temporal coding can involve different mechanisms of synaptic signal transmission. Also, current findings (Castro-Almanancos \& Connors, 1996) showed that repetitive stimuli can influence synaptic strengthening by long-term potentiation of synapses.

Temporal coding becomes more robust with experience, and drives synaptic plasticity in cortical networks (Hestrin \& Galarreta, 2005). A candidate mechanism for facilitating temporal coding at the macroscopic level is oscillatory brain activity such as the hippocampal theta rhythm (Buzsáki \& Draguhn, 2004; Siapas, Lubenov \& Wilson, 2005). Human neuronal networks display oscillations in a broad frequency range from approximately 0.05 to $500 \mathrm{~Hz}$, and may represent codes for temporal order information and binding (Buzsáki \& Draguhn, 2004). An important characteristic of such mechanisms is synchronization, in general, and phase synchronization among temporally linked processes, in particular (Llinas, Urbano, Leznik, Ramirez \& van Marle, 2005).

We contend that the two measures that are more sensitive to phase information, that is, PLI and PC, can be regarded as indicators of temporal coding in the context of this study. Conversely, the two power measures are more indicative of rate coding, especially when PLI and $\mathrm{PC}$ are low. Based on these premises, the lifespan dissociation in temporal dynamics of oscillatory activity observed in this study may point to lifespan changes in coding mechanisms at the cellular level. Specifically, children may predominantly use a rate code for representing sensory events during auditory perception, whereas adults may predominantly use temporal coding.

Rate coding is known to be present early in ontogeny, whereas temporal coding is known to emerge at later stages of development and learning (Feldman, 2000). Thus, the immaturity of inhibitory mechanisms (Dempster, 1992) may have decreased the likelihood of the use of stimulus-related phase-locking as a prevalent coding strategy in children. It is worth noting that younger and older children did not differ from each other in the PLI, but that older children showed significantly higher PC than young children. In other words, while local phase synchronization did not differ between the two groups, phase synchronization across electrodes, or large-scale synchronization, was higher in OC than in YC. Presumably, these childhood transitions reflect the continuing maturation of cortico-cortical connections, myelination processes, and synaptic density changes in prefrontal cortex and other cortical areas (Courchesne, 1990). Synaptic density in humans increases up to 5-8 years of age and then decreases dramatically during early adolescence (Huttenlocher \& Dabholkar, 1997). These changes in cortical architecture, called pruning, seem to play a crucial role in maturation of neural connectivity and may be the origin of differences in large-scale synchronization between $\mathrm{YC}$ and $\mathrm{OC}$.

Also of interest are the observed similarities and differences between young and older adults. Relative to children, both young and older adults made prevalent use of temporal coding, with older adults showing even higher phase synchronization at theta and alpha frequency during the first time interval as well as lower EP and WP than younger adults. This trend defies the idea, first suggested by Ribot (1882), that old adults regress to ontogenetically early neuronal and behavioral repertoires (e.g. 'first-in, last-out principle'; cf. Bäckman, Small, Wahlin \& Larsson, 2000). At the same time, stimulus-locked synchronization measures (i.e. PLI and EP), that were positively correlated with perceptual speed (IP scores) in younger adults, especially under the attended condition, showed negative correlations to independently assessed measures of perceptual speed under the attended condition (see Figures 4 and 5) and no reliable correlations under unattended conditions in older adults. Such negative synchronization-speed correlations were found for theta and alpha frequencies and relatively early after stimulus onset; in younger adults, positive synchronization-speed correlations were found for theta and especially delta frequencies, and for later, presumably more cognitive, stages of processing. Again, in most cases, differences in correlations between young and older adults were statistically significant.

Given their negative relation to perceptual speed, and in view of curvilinear lifespan gradients in inhibitory functioning reported elsewhere (Bedard, Nichols, Barbosa, Schachar, Logan \& Tannock, 2002; Kinsbourne \& Hicks, 1978; Volkow, Gur, Wang, Fowler, Moberg, Ding, Hitzemann, Smith \& Logan, 1998), the high levels 
of early phase locking among older adults are unlikely to reflect a further increase in inhibitory efficiency from young adulthood to old age. Rather, the negative correlations between early synchronization and perceptual speed among older adults suggest that the early representation of sensory events in old age may occur in a highly stimulus-driven (e.g. automatic) manner that is less easily modulated by top-down influences and less easily integrated into ongoing cognitive activity. In agreement with this line of reasoning, the amount of stimulus-related synchronization as assessed by PLI and EP varied less as a function of task conditions (attended versus unattended, deviant versus standard) in older adults than in younger adults. At a more general level, this interpretation is consistent with a dedifferentiation view of cognitive aging ( $\mathrm{Li} \&$ Lindenberger, 1999; Lindenberger \& Baltes, 1994; Park et al., 2004), and with recent behavioral findings demonstrating that older adults are greatly impaired in disregarding task-irrelevant environmental cues (Spieler, Mayr \& LaGrone, 2006).

In sum, the present study shows that the perceptual representation of auditory events undergoes profound changes from childhood to adulthood and old age. We speculate that these changes reflect a decreasing reliance on rate coding, and an increasing reliance on temporal coding. This speculation needs to be corroborated (or refuted) by further examining age differences in the task-dependent modulation of background activity and phase locking. If confirmed, future work needs to address the mechanisms, the reversibility, and the generality of the transition from rate coding to temporal coding during different periods of the human lifespan. In particular, more needs to be known about the ways in which representational formats of sensory events can change profoundly without disrupting their integration into other aspects of information processing, such as conscious experience. For instance, Lu, Liang and Wang (2001) showed that two largely distinct populations of neurons in the auditory cortex of awake primates with stimulus-synchronized and non-stimulus-synchronized discharges appear to represent slowly and rapidly occurring auditory events by use of temporal and rate codes, respectively. Thus, processing of temporal information may be achieved by combinations of temporal and rate coding. How the relation between the two code mechanisms changes with age remains to be understood in greater detail.

Finally, some limitations of the present study need to be acknowledged. First, due to the modest sample size, the present analyses may lack power to detect existing group differences in synchronization. Second, the observed differences between children and adults, especially in the spectral power measures, can be influenced by differences in skull thickness (e.g. Pfefferbaum, 1990). However, it is unlikely that age differences in skull thickness would fully account for age differences in power. In addition, it is hard to conceive how differences in skull thickness would result in age differences in observed synchronization.

\section{Acknowledgements}

This study was supported by the German Research Foundation, DFG Research Unit, 'Binding: Functional Architecture, Neuronal Correlates and Ontogenesis'. The authors are grateful to Roland Benoit, Yvonne Brehmer, Stephan Glied, Esther Runkel, Myriam Sander, Zurab Schera, Gundula Stoll and Benjamin Straube for technical assistance and for carrying out the experimental part of the study. We also thank Penny Motley and Julia Delius for editorial assistance.

\section{References}

Abbott, L.F., \& Regehr, W.G. (2004). Synaptic computation. Nature, 431, 796-803.

Adrian, E.D. (1926). The impulses produced by sensory nerve endings. Part I. Journal of Physiology (London), 6, 49-72.

Adrian, E.D., \& Bronk, D.W. (1929). The discharge of impulses in motor nerve fibres. Part II. Journal of Physiology (London), 67, 119-151.

Bäckman, L., Small, B.J., Wahlin, A., \& Larsson, M. (2000). Cognitive functioning in very old age. In F.I.M. Craik \& T.A. Salthouse (Eds.), The handbook of aging and cognition (2nd edn., pp. 499-558). Mahwah, NJ: Erlbaum.

Baltes, P.B., \& Lindenberger, U. (1997). Emergence of a powerful connection between sensory and cognitive functions across the adult life span: a new window to the study of cognitive aging? Psychology and Aging, 12, 12-21.

Baltes, P.B., \& Mayer, K.U.(Eds.)(1999). The Berlin Aging Study: Aging from 70 to 100. New York: Cambridge University Press.

Bastiaansen, M., \& Hagoort, P. (2003). Event-induced theta responses as a window on the dynamics of memory. Cortex, 39, 967-992.

Bedard, A.-C., Nichols, S., Barbosa, J.A., Schachar, R., Logan, G.D., \& Tannock, R. (2002). The development of selective inhibitory control across the life span. Developmental Neuropsychology, 21, 93-111.

Buzsáki, G. (2005). Theta rhythm of navigation: link between path integration and landmark navigation, episodic and semantic memory. Hippocampus, 15, 827-840.

Buzsáki, G., \& Draguhn, A. (2004). Neural oscillations in cortical networks. Science, 304, 1926-1929.

Cacace, A.T., \& McFarland, D.J. (2003). Spectral dynamics of electroencephalographic activity during auditory information processing. Hearing Research, 176, 25-41.

Carhart, R., \& Jerger, J.F. (1959). Preferred method for clinical determination of pure-tone thresholds. Journal of Speech and Hearing Disorders, 24, 330-345.

Castro-Alamancos, M.A., \& Connors, B.W. (1996). Short-term synaptic enhancement and long-term potentiation in neocortex. Proceedings of the National Academy of Sciences USA, 93, 1335-1339.

Courchesne, E. (1990). Chronology of postnatal human brain development: event-related potential, positron emission tomography, myelinogenesis and synaptogenesis studies. In J.W.P. Rohrbaugh \& R. Johnson (Eds.), Event-related brain potentials (pp. 210-241). New York: Oxford University Press.

Dempster, F.N. (1992). The rise and fall of the inhibitory mechanism: toward a unified theory of cognitive development and aging. Developmental Review, 12, 45-75. 
Destexhe, A., \& Marder, E. (2004). Plasticity in single neuron and circuit computations. Nature, 431, 789-795.

Duffy, F.H., McAnulty, G.B., \& Albert, M.S. (1993). The pattern of age related differences in electrophysiological activity of healthy males and females. Neurobiology of Aging, 14, 73-84.

Feldman, D.E. (2000). Inhibition and plasticity. Nature Neuroscience, 3, 303-304.

Gratton, G., Coles, M.G., \& Donchin, E. (1983). A new method for off-line removal of ocular artifact. Electroencephalography and Clinical Neurophysiology, 55, 468-484.

Gruber, W.R., Klimesch, W., Sauseng, P., \& Doppelmayr, M. (2005). Alpha phase synchronization predicts P1 and N1 latency and amplitude size. Cerebral Cortex, 15, 371-377.

Harmony, T., Fernandez, T., Silva, J., Bernal, J., Diaz-Comas, L., Reyes, A., Marosi, E., Rodriguez, M., \& Rodriguez, M. (1996). EEG delta activity: an indicator of attention to internal processing during performance of mental tasks. International Journal of Psychophysiology, 24, 161-171.

Hestrin, S., \& Galarreta, M. (2005). Synchronous versus asynchronous transmitter release: a tale of two types of inhibitory neurons. Nature Neuroscience, 8, 1283-1284.

Huttenlocher, P.R., \& Dabholkar, A.S. (1997). Regional differences in synaptogenesis in human cerebral cortex. Journal of Comparative Neurology, 387, 167-178.

Karrasch, M., Krause, C.M., Laine, M., Lang, A.H., \& Lehto, M. (1998). Event-related desynchronization and synchronization during an auditory lexical matching task. Electroencephalography and Clinical Neurophysiology, 107, 112-121.

Kinsbourne, M., \& Hicks, R.E. (1978). Functional cerebral space: a model for overflow, transfer and interference effects in human performance. A tutorial review. In J. Requin (Ed.), Attention and performance VII (pp. 345-362). Hillsdale, NJ: Erlbaum.

Klimesch, W., Schack, B., Schabus, M., Doppelmayr, M., Gruber, W., \& Sauseng, P. (2004). Phase locked alpha and theta oscillations generate the P1-N1 complex and are related to memory performance. Cognitive Brain Research, 19, 302-316.

Krause, C.M., Lang, A.H., Laine, M., Kuuisto, M., \& Pörn, B. (1996). Event-related EEG desynchronization and synchronization during an auditory memory task. Electroencephalography and Clinical Neurophysiology, 98, 319-326.

Lachaux, J.-P., Rodriguez, E., van Quyen, M.L., Lutz, A., Martinerie, J., \& Varela, F.J. (2000). Studying single-trials of phase synchronous activity in the brain. International Journal of Bifurcation and Chaos, 10, 2429-2439.

Li, S.-C., \& Lindenberger, U. (1999). Cross-level unification: a computational exploration of the link between deterioration of neurotransmitter systems and dedifferentiation of cognitive abilities in old age. In L.G. Nilsson \& H. Markowitsch (Eds.), Cognitive neuroscience of memory (pp. 103-146). Toronto: Hogrefe.

Li, S.-C., Lindenberger, U., \& Sikström, S. (2001). Aging cognition: from neuromodulation to representation to cognition. Trends in Cognitive Sciences, 5, 479-486.

Lindenberger, U., \& Baltes, P.B. (1994). Sensory functioning and intelligence in old age: a strong connection. Psychology and Aging, 9, 339-355.

Lindenberger, U., Mayr, U., \& Kliegl, R. (1993). Speed and intelligence in old age. Psychology and Aging, 8, 207-220.

Llinas, R., Urbano, F.J., Leznik, E., Ramirez, R.R., \& van Marle, H.J.F. (2005). Rhythmic and dysrhythmic thalamo- cortical dynamics: GABA systems and the edge effect. Trends in Neurosciences, 28, 325-333.

Lu, T., Liang, L., \& Wang, X. (2001). Temporal and rate representations of time-varying signals in the auditory cortex of awake primates. Nature Neuroscience, 4, 1131-1138.

McAllister, A.K., Katz, L.C., \& Lo, D.C. (1999). Neurotrophins and synaptic plasticity. Annual Review of Neuroscience, 22, 295-318.

McEvoy, L.K., Pellouchoud, E., Smith, M.E., \& Gevins, A. (2001). Neurophysiological signals of working memory in normal aging. Cognitive Brain Research, 11, 363-376.

Makeig, S., Westerfield, M., Jung, T.-P., Enghoff, S., Townsend, J., Courchesne, E., \& Sejnowski, T.J. (2002). Dynamic brain sources of visual evoked responses. Science, 295, 690-694.

Marciani, M.G., Maschio, M., Spanedda, F., Caltagirone, C., Gigli, G.L., \& Bernardi, G. (1994). Quantitative EEG evaluation in normal elderly subjects during mental processes: age related changes. International Journal of Neuroscience, $\mathbf{7 6}$, 131-140.

Mehta, M.R., Lee, A.K., \& Wilson, M.A. (2002). Role of experience and oscillations in transforming a rate code into a temporal code. Nature, 417, 741-746.

Mueller, V., Brehmer, Y., von Oertzen, T., Li, S.-C., \& Lindenberger, U. (2008). Electrophysiological correlates of selective attention: a lifespan comparison. BMC Neuroscience, 9, 18.

Nelson, C.A., \& Luciana, M. (Eds.) (2001). Handbook of developmental cognitive neuroscience. Cambridge, MA: MIT Press.

Nunez, P.L. (1995). Neocortical dynamics and human EEG rhythms. Oxford: Oxford University Press.

Oken, B.S., \& Kaye, J.A. (1992). Electrophysiological function in the healthy, extremely old. Neurology, 42, 519-526.

Park, D.C., Polk, T.A., Park, R., Minear, M., Savage, A., \& Smith, M.R. (2004). Aging reduces neural specialization in ventral visual cortex. Proceedings of the National Academy of Sciences, USA, 101, 13091-13095.

Pfefferbaum, A. (1990). Model estimates of CSF and skull influences on scalp-recorded ERPs. Alcohol, 7, 479-482.

Pfurtscheller, G., \& Lopes da Silva, F.H. (1999). Event-related EEG/MEG synchronization and desynchronization: basic principles. Clinical Neurophysiology, 110, 1842-1857.

Polich J. (1996). Meta-analysis of P300 normative aging studies. Psychophysiology, 33, 334-353.

Polich, J. (1997). EEG and ERP assessment of normal aging. Electroencephalography and Clinical Neurophysiology, 104, 244-256.

Ribot, T. (1882). Diseases of memory. New York: Appleton.

Siapas, A.G., Lubenov, E.V., \& Wilson, M.A. (2005). Prefrontal phase locking to hippocampal theta oscillations. Neuron, 46, 141-151.

Simoes, C., Jensen, O., Parkkonen, L., \& Hari, R. (2003). Phase locking between human primary and secondary somatosensory cortices. Proceedings of the National Academy of Sciences, USA, 100, 2691-2694.

Spieler, D.H., Mayr, U., \& LaGrone, S. (2006). Outsourcing cognitive control to the environment: adult age differences in the use of task cues. Psychonomic Bulletin \& Review, 13, 787-793.

Srinivasan, R., Russell, D.P., Edelman, G.M., \& Tononi, G. (1999). Increased synchronization of neuromagnetic responses during conscious perception. Journal of Neuroscience, 19, $5435-5448$. 
Stein, R.B., Gossen, E.R., \& Jones, K.E. (2005). Neuronal variability: noise or part of the signal? Nature Reviews Neuroscience, 6, 389-397.

Tallon-Baudry, C., \& Bertrand, O. (1999). Oscillatory gamma activity in humans and its role in object representation. Trends in Cognitive Sciences, 3, 151-162.

Varela, F., Lachaux, J.-P., Rodriguez, E., \& Martinerie, J. (2001). The brainweb: phase synchronization and large-scale integration. Nature Reviews Neuroscience, 2, 229-239.

Volkow, N.D., Gur, R.C., Wang, G-J., Fowler, J.S., Moberg, P.J., Ding, Y.-S., Hitzemann, R., Smith, G., \& Logan, J. (1998). Association between decline in brain dopamine activity with age and cognitive and motor impairment in healthy individuals. American Journal of Psychiatry, 155, 344-349.

Yordanova, J., \& Kolev, V. (1997). Alpha response system in children: changes with age. International Journal of Psychophysiology, 26, 411-430.

Yordanova, J., \& Kolev, V. (1998). Developmental changes in the theta response system: a single sweep analysis. Journal of Psychophysiology, 12, 113-126.

Received: 15 November 2007

Accepted: 18 July 2008 